\title{
Differences in In Vitro Dissolution Rates Using Single-Point and Multi-Point Sampling
}

\author{
Limin Zhang ${ }^{1,3}$, Khanh Ha', Brent Kleintop', Shannon Phillips², \\ Barry Scheer ${ }^{1}$ \\ 'Analytical Research \& Development, Bristol-Myers Squibb Pharmaceutical Research Institute, \\ New Brunswick, NJ. \\ ${ }^{2}$ The Dow Chemical Company, Texas Operations, Freeport, TX.
}

\section{INTRODUCTION}

$\mathrm{n}$ vitro dissolution testing is an important physicochemical tool used to measure drug release rates during both early and late stages of drug development. In both cases, a product-specific discriminating dissolution method is utilized as a quality control tool to evaluate batch-to-batch consistency and as a predictive means to gain a post-market bio-waiver in cases where there is in vitro-in vivo correlation or in vitro-in vivo relation. Developing rugged methods with appropriate discriminatory dissolution parameters is often a challenging objective regardless of the solubility of the compounds. Method development is further challenged when hydrodynamic effects within the vessel (1-4) impact method ruggedness. The hydrodynamic environment within the vessel can be affected by many variables including the position of the tablet $(5,6)$ and the presence and position of a measuring (7) or sampling probe (8). Thus, for poorly soluble drugs, it is challenging to develop an appropriate dissolution method that is rugged, discriminating, and maintains sufficient sink conditions.

While implementing a dissolution method in a manufacturing quality control (QC) laboratory, we encountered a unique method ruggedness issue. Previously, we successfully transferred the validated discriminating dissolution method from our R\&D facility to both a stability laboratory and a manufacturing QC laboratory. However, during the manufacturing validation campaign, several tablet batches exhibited slower dissolution rates in the QC laboratory when compared with the historical dissolution rates generated in the R\&D and stability laboratories. Several of the process validation batches were at risk of proposed specification failure, which had required Stage II $\left(\mathrm{S}_{2}\right)$ testing. An investigation was initiated to determine if the root cause of the low results was due to a method robustness or product quality issue.

The drug product that exhibited this behavior was prepared using a BCS class II (low solubility and high permeability) compound in which dissolution was the

${ }^{3}$ Corresponding author. rate-limiting step for the in vivo absorption. During development and stability assessment of the drug product, in vitro drug release was routinely evaluated using a typical dissolution profile obtained by sampling at several discrete time points. Batch-to-batch variability was assessed by determining the statistical difference between the dissolution profiles of each batch ( $f_{2}$ similarity test). The best performing (most discriminatory) batches against which the other batches were compared, were designated based on the dissolution profiles of prototypes with different types of granulation, type or amount of excipients, and batch chemical stability. During the process validation campaign, dissolution testing was conducted using a single sample pull at one specified time point to meet an acceptance criterion for batch release. Since some of the batches required $S_{2}$ testing, an initial investigation was focused on the effects that sampling technique had on the hydrodynamics of the dissolution vessel, such as manual versus automated sampling, or single-point versus multi-point profiling. In addition, other method parameters such as paddle rotation speed and media $\mathrm{pH}$ were evaluated as part of this investigation.

\section{EXPERIMENTAL}

This investigation was performed on a tablet dosage form containing a weakly basic BCS Class II (low solubility and high permeability) drug compound, with a $\mathrm{pK}_{\mathrm{a}}$ value of 3.6. All batches listed in this investigation were made from the same or similar formulation and manufacture processes.

The dissolution method under investigation employed compendia conditions with USP Apparatus 2 (paddle) at a rotation speed of $50 \mathrm{rpm}$ in a medium of $50 \mathrm{mM}, \mathrm{pH} 6.8$ phosphate buffer. The discriminatory ability of these method conditions was agreed upon after an FDA-interface meeting prior to filing the NDA for this product.

The time points for multi-point (profile) sampling used to support all phases of clinical development and stability were 10,20,30, 45, and $60 \mathrm{~min}$. Single-point sampling at 30 min was used for release testing in the QC laboratory. Quantitation of the dissolution samples was performed by HPLC with UV detection. 
The automated dissolution method used to support all phases of clinical development, technology transfer, clinical stability, and registration stability utilized a Multidose ${ }^{\circledR}$ G3 manufactured by Caliper Science, Inc. This automated system can perform several unattended cycles of media dispensing, temperature equilibration, vessel filling, temperature reading, sample drop, dissolution, sampling, filtration, sample dispensing, and vessel washing.

\section{RESULTS AND DISCUSSION}

The registration dissolution method employed a dissolution medium of $\mathrm{pH} 6.8$ based on the physiologic $\mathrm{pH}$, sink condition, and acceptable solubility. As shown in the aqueous solubility curve for the subject drug compound (Figure 1), the solubility changes significantly within the $\mathrm{pH}$ range of $6.5-7.2$, suggesting that small changes in the dissolution media $\mathrm{pH}$ within this range could lead to significant differences in the dissolution profile as sink conditions are approached. At the $\mathrm{pH}$ of 6.8 used in the method, the solubility of the active drug is $44 \mu \mathrm{g} / \mathrm{mL}$. For a tablet with $2.5-\mathrm{mg}$ label strength, the dissolution concentration $(2.5 \mu \mathrm{g} / \mathrm{mL})$ corresponds to a sink condition that is 17 times the saturation volume.

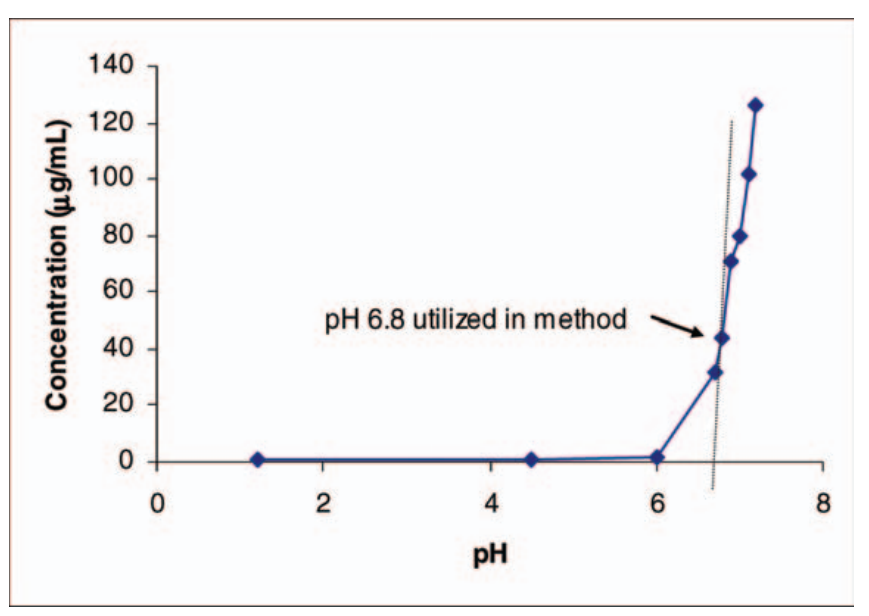

Figure 1.pH-Solubility profile for the subject drug compound (dashed line indicates the $\mathrm{pH}$ utilized by the method). Aqueous solubility at $37^{\circ} \mathrm{C}$ is $32 \mu \mathrm{g} / \mathrm{mL}$ at $\mathrm{pH} 6.7,44 \mu \mathrm{g} / \mathrm{mL}$ at $\mathrm{pH} 6.8$, and $71 \mu \mathrm{g} / \mathrm{mL}$ at $\mathrm{pH} 6.9$, respectively.
However, since solubility is a primary driver for the dissolution of this drug substance, method robustness could be affected by several factors such as $\mathrm{pH}$ or hydrodynamic disturbances.

During validation of the manufacturing process for this tablet product, dissolution testing of batches was performed by the QC laboratory using automated single-point sampling at the 30 -min time point. As shown in Table 1, several batches exhibited low dissolution rates that failed the Stage I specification of $80 \%(\mathrm{Q})$ dissolved in 30 min, resulting in the need to initiate $S_{2}$ testing. Understanding the root cause of these low results became essential since it could be indicative of a method ruggedness or product quality issue. Additionally, it is undesirable to incur a high frequency of $S_{2}$ testing from a product quality, manufacturing process robustness, and operational efficiency perspective.

During the investigation of the $S_{1}$ dissolution failures, the tablet batches were reanalyzed by the R\&D laboratory using an automated profile method. As shown in Table 2, the R\&D laboratory dissolution results were $6-7 \%$ higher than those generated in the QC laboratory using the automated single-point method. These higher results were consistent with our previous development experience. This discrepancy between the R\&D and QC laboratories was not observed during the interlaboratory method transfer, where profile sampling was performed in both laboratories (Table 3). Because the sampling technique (single-point versus multi-point profiling) was the only major difference in the procedure used by the two laboratories, sampling effects became the main focus of subsequent investigations.

\section{Comparison of Dissolution Data Using Single-Point versus Multi-Point Sampling}

A comparison of single-point and multi-point sampling across different automated dissolution systems from multiple laboratories showed a consistent 3-5\% bias (Table 4). The consistency of the bias suggested that the observed difference was inherent to the method parameters and was not a function of the instrument, site, and analyst. It further suggested that the automated dissolution systems were functioning properly across all laboratories and that method reproducibility was not an issue.

Table 1. Dissolution Results for Process Validation Batches Using Single-Point Sampling.

Percent of Label Dissolved at $30 \mathrm{~min}$ *

\begin{tabular}{|c|c|c|c|c|c|c|c|}
\hline & Tablet \#1 & Tablet \#2 & Tablet \#3 & Tablet \#4 & Tablet \#5 & Tablet \#6 & Average \\
\hline Batch 1 & 90 & 87 & 88 & 83 & 85 & 85 & 86 \\
\hline Batch 2 & 87 & 88 & 88 & 86 & 88 & 82 & 87 \\
\hline Batch 3 & 87 & 87 & 86 & 85 & 86 & 83 & 86 \\
\hline
\end{tabular}

* Specification: Not less than $80 \%(\mathrm{Q})$ of label dissolved in $30 \mathrm{~min}$.

Figures in bold represent results that failed the proposed specification of $80 \%(Q)+5 \%$ at Stage I. 
Table 2. Comparison of Dissolution Results from Process Validation Batches Between AR\&D (Multi-Point Sampling) and QC Laboratories (Single-Point Sampling).

Percent of Label Dissolved at $30 \min (n=12)$

\begin{tabular}{lccc} 
& Average (AR\&D) & Average (QC) & Difference \\
\hline Batch 1 & 93 & 86 & 7 \\
\hline Batch 2 & 93 & 87 & 6 \\
\hline Batch 3 & 93 & 86 & 7 \\
\hline
\end{tabular}

Table 3. Dissolution Data Generated During Method Technology Transfer Using Automated System.

Average Percent of Label Dissolved $(n=12)$ *

\begin{tabular}{lccc} 
Time Point (min) & $\begin{array}{c}\text { Transferring Lab } \\
\text { (AR\&D) }\end{array}$ & $\begin{array}{c}\text { Receiving Lab } \\
\text { \#1 (QC) }\end{array}$ & $\begin{array}{c}\text { Receiving Lab \#2 } \\
\text { (Stability) }\end{array}$ \\
\hline 10 & 80 & 81 & 82 \\
\hline 20 & 94 & 93 & 92 \\
\hline 30 & 96 & 95 & 94 \\
\hline 45 & 98 & 96 & 96 \\
\hline 60 & 99 & 96 & 96
\end{tabular}

* Only profile sampling was performed during method transfer. Results from all labs were within acceptance criteria. Results in bold represent the results of 30-min time point. A representative clinical batch was used for technology transfer.

Table 4. Comparison of Single-Point and Profile Dissolution Results Obtained Using Multiple Automated Systems.

Average Percent of Label Dissolved at $30 \min (n=12)$

Automated

System \# Single-Point Sampling Multi-Point Sampling Difference

\begin{tabular}{llll}
\hline 1 & 89 & 94 & 5 \\
\hline 2 & 93 & 96 & 3 \\
\hline 3 & 86 & 91 & 5 \\
\hline
\end{tabular}

* A representative clinical batch was used.

\section{Re-Evaluation of the Validation Parameters of the Automated Dissolution Method}

The slower dissolution rates were observed with both the manual and automated methodologies when single-point sampling was performed, as shown in Figure 2. For thoroughness, we explored several potential sources of sample loss due to the unique features of the automated system versus the manual system sampling. Tubing flush volume and filter loading were among the validation parameters that were reevaluated, since they could potentially contribute to loss of drug. Results indicated that these sampling parameters were properly

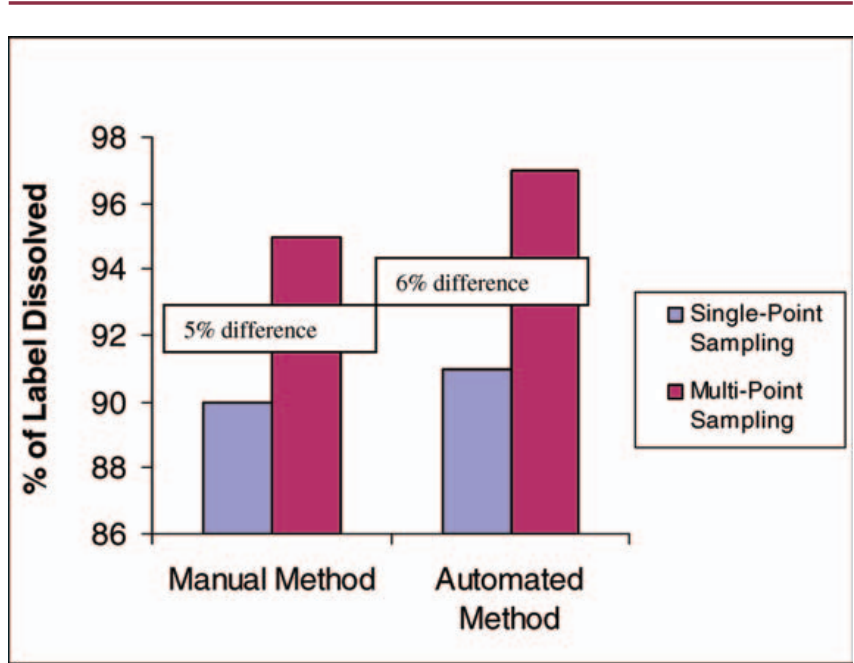

Figure 2. Comparison of results obtained for single-point and multi-point sampling at 30-min time point on a representative clinical release batch. A 5-6\% faster release was obtained when using multi-point sampling.

validated and did not contribute significantly to any drug loss.

\section{Effect of Sampling Probe}

The automated sampling mechanism consisted of a sample pump capable of draw speeds from 5 to $12 \mathrm{~mL} / \mathrm{min}$. The sampling probe has an outer diameter of approximately $0.25 \mathrm{in}$. and a length of $9 \mathrm{in}$. The sampling position was automatically adjusted to meet USP requirements for a particular medium volume. In order to eliminate sample carryover, a sample volume of $16 \mathrm{~mL}$ was required. To collect this volume with the pump set at the maximum speed of $12 \mathrm{~mL} / \mathrm{min}$, the sampling probe was immersed in the dissolution medium for approximately 90 s during each sampling interval.

During multi-point sampling, prior to the 30-min time point, the probe was immersed in the dissolution medium at each of the 10-min and 20-min time points for a period of $90 \mathrm{~s}$. Although relatively brief, this probe immersion time could potentially cause disruption of currents within the vessel and introduce turbulence in the flow. During single-point sampling, the probe is not in contact with the medium until it withdraws the single sample at the 30-min time point. The profile sample withdrawals increase disruption of the hydrodynamic flow in the dissolution vessel, resulting in faster release of the drug at the 30-min time point as compared with single-point sampling.

To determine the hydrodynamic disturbance introduced by multi-point sampling as compared with single-point sampling, it would be ideal to simulate the automated multi-point sampling by immersing the probe in the vessel without sampling for the 10-min and 20-min time points. Due to the limitation of the software, the automated dissolution system does not have the capability to sample this way. Instead, we performed a 
single-point dissolution experiment where the probe was immersed in the dissolution vessel for the entire $30 \mathrm{~min}$ prior to sampling at the 30-min time point to determine the maximum effect this hydrodynamic disturbance would produce. If the hydrodynamic effect caused by the sampling probe was indeed the root cause of the discrepancy between single-point and profile sampling, this experiment would simulate the maximum difference that would be expected due to the presence of the sampling probe. Results in Table 5 demonstrate that at the 30-min time point, the percent drug released when the probe was immersed in the media for the entire $30 \mathrm{~min}$ was $6 \%$ greater than when the probe was not immersed in the media prior to the sampling at $30 \mathrm{~min}$. This difference is similar to the differences observed in the previous comparison experiments, as shown in Table 2. These results suggest that the presence of the sampling probe in the media had a significant impact on the hydrodynamic environment and likely contributed to the differences in dissolution results obtained between single-point and multi-point sampling.

This hydrodynamic effect was also evaluated using manual sampling for single-point and profile tests. These manual results show a similar trend when compared with automated systems (Figure 2). This trend is evident even though the cannula immersion time was much shorter for the manual method and the cannula (outer diameter of approximately $0.125 \mathrm{in}$.) is smaller than the automated probe. Since the same trend was observed in the manual system, the method appears highly susceptible to hydrodynamic effects.

\section{Evaluation of Paddle Speed and pH}

To further evaluate dissolution parameters that minimize hydrodynamic effects but still provide sufficient discrimatory power, experiments were conducted using different paddle speeds and media $\mathrm{pH}$. The initial dissolution method submitted with the IND for this product employed a pH 7.0 phosphate buffer and 60-rpm paddle rotation speed. Due to the higher solubility of the drug at $\mathrm{pH} 7.0$ and the greater agitation, this method provided a more rapid and robust release than the compendial method conditions. The results, summarized in Table 6, demonstrate that the hydrodynamic effects are minimized when higher rotation speeds and improved sink conditions (such as increased $\mathrm{pH}$ ) are applied,

Table 5. Comparison of Single-Point Results Obtained Using Different Sampling Strategies.

Average Percent of Label Dissolved at $30 \mathrm{~min}(\mathrm{n}=12)^{*}$

Probe immersed in the Probe immersed in the vessel vessel for entire $30 \mathrm{~min}$ only at the 30 -min time point Difference

\begin{tabular}{lll}
\hline 94 & 88 & 6 \\
\hline
\end{tabular}

* A representative clinical batch was used.

Dissolution Technologies | NOVEMBER 2007
Table 6. Comparison of Dissolution Results for Single-Point Versus Profile Sampling Using Altered Media pH and Paddle Rotation Speeds.

\begin{tabular}{lcccc}
\hline & & \multicolumn{3}{c}{ Average Percent of Label Dissolved } \\
at 30 in $^{*}$ & \\
Medium pH & Paddle Speed & $\begin{array}{c}\text { Single-Point } \\
\text { Sampling }\end{array}$ & $\begin{array}{c}\text { Multi-Point } \\
\text { Sampling }\end{array}$ & Difference \\
\hline 6.8 & 50 RPM & $86-93$ & $91-96$ & $3-5$ \\
\hline 6.8 & 60 RPM & 94 & 95 & 1 \\
\hline 7.0 & 50 RPM & 94 & 96 & 2 \\
\hline 7.0 & 60 RPM & 95 & 96 & 1 \\
\hline * A representative clinical batch was used & &
\end{tabular}

regardless of the sampling technique. These conditions create a more robust dissolution method. Furthermore, the more robust dissolution method is able to discriminate the batches based on the amount of disintegrant used in the formulation, as shown in Figure 3.

\section{CONCLUSIONS}

Here we provide an example where hydrodynamic effects can potentially influence in vitro dissolution results. When developing a discriminating and robust dissolution method, one should evaluate how the method will ultimately be implemented in the manufacturing environment. A seemingly robust and discriminating method can present unexpected results based on the number of sampling time points and sample withdrawals. In the case of this particular drug product, it was determined that multiple sample pulls from the dissolution vessel, as in a "profile" experiment (typically used in the R\&D or stability testing environments) versus a

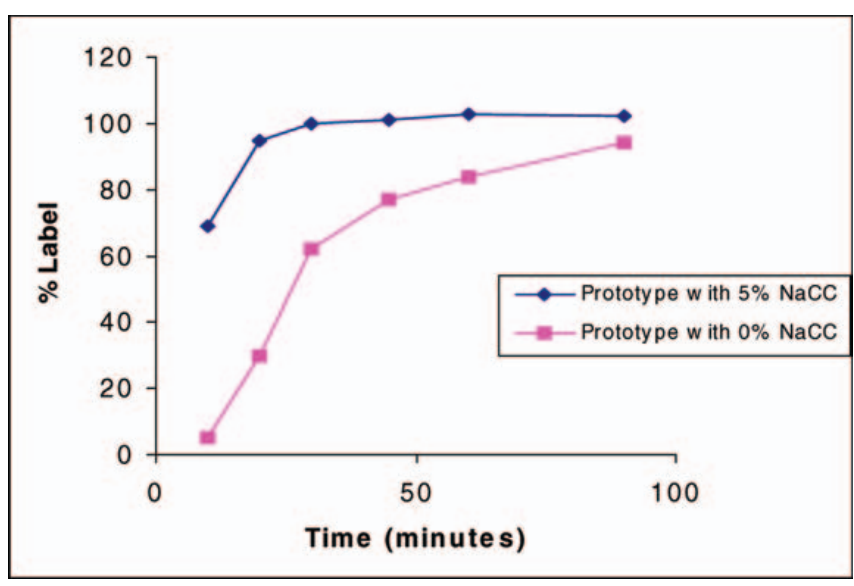

Figure 3. Dissolution profiles of two prototype formulations using altered medium $\mathrm{pH}(\mathrm{pH} 7.0)$ and paddle rotation speed (60 rpm). Prototype formulations containing $0 \%$ and $5 \%$ sodium croscarmellose (NaCC) were evaluated. 
single-pull (typically used in the QC environment), resulted in different dissolution rates. It is believed that this discrepancy is a result of a greater disturbance of the fluid hydrodynamics in the dissolution vessel caused by the additional insertions and residence of the sampling probe in the vessel during multi-point sampling. Additional examples in the literature suggest that the hydrodynamic conditions, drug release pattern, or mechanical forces of an in vitro dissolution are crucial to the drug release rate $(9,10)$. In the case of this dissolution method, the impact of the sampling technique on fluid hydrodynamics and the subsequent effect on method robustness should not be overlooked. We initially proposed a dissolution method that employed a rotation speed of $60 \mathrm{rpm}$ and a medium $\mathrm{pH}$ of 6.8 for use during registration stability. Based on the $\mathrm{f}_{2}$ calculation, these proposed parameters did not result in significantly different dissolution profiles as compared with the compendia conditions of 50-rpm paddle speed with a pH 6.8 medium. We chose to utilize the 50-rpm paddle speed to be in alignment with the regulatory agency suggestion. However, the hydrodynamic effects caused by sampling were not evaluated, and the revised method was found to be more sensitive to subtle changes in the dissolution conditions, such as sampling over multiple time points. Use of single-point versus multi-point profile testing should be taken into account early in the method development process. Technology transfer using single-point sampling is critical. Based on the lessons learned on this project, dissolution methods for other drug products in development have been evaluated for method robustness under different sampling conditions. Differences in percent label dissolved between single-point sampling and profile sampling were observed for other methods, and investigations were performed to minimize the problems. The results of this investigation have influenced how we conduct dissolution method development.

\section{ACKNOWLEDGMENT}

The authors wish to acknowledge the insightful discussions provided by Vishwas Nesarikar, Robert Perrone, Scott Jennings, Ruben Lozano, Thomas Raglione, Pankaj
Shah, Shufang Niu, and other members of our research groups here at Bristol-Myers Squibb.

\section{REFERENCES}

1. Underwood, F.; Cadwallder, D. Effects of various hydrodynamic conditions on dissolution rate determination.J.Pharm. Sci. 1976, 65, 697.

2. Baxter, J. L.; Kukura, J.; Muzzio, F. J. Hydrodynamic-induced variability in the USP Apparatus II dissolution test. Int. J. Pharm. 2005, 292, 17-28.

3. Wu, Y.; Kildsig, D. O.; Ghaly, E. S. Effect of hydrodynamic environment on tablets dissolution rate. Pharm. Dev. Technol. 2004, 9 (1), 25-37.

4. McCarthy, L. G.; Bradley, G.; Sexton, J. C.; Corrigan, O. I.; Healy, A. M. Computational fluid dynamic modeling of the paddle dissolution apparatus: agitation rate, mixing patterns, and fluid velocities. AAPS PharmSciTech 2004, 5 (2), Article 31.

5. D'Arcy, D. M.; Corrigan, O. I.; Healy, A. M. Hydrodynamic simulation of asymmetrically positioned tablets in the paddle dissolution apparatus: impact on dissolution rate and variability. J. Pharm. Pharmacol. 2005, 57 (10), 1243-1250.

6. Healy, A. M.; McCarthy, L. G.; Gallagher, K. M.; Corrigan, O.I. Sensitivity of dissolution rate to location in the paddle dissolution apparatus. J. Pharm. Pharmacol. 2002, 54, 441.

7. Lu, X.; Lozano, R.; Shah, P. A. In situ dissolution testing using different UV fiber optic probes and instruments. Dissolution Technol. 2003, 10 (4), 6-15.

8. Savage, T. S.; Wells, C. E. Automated sampling of in vitro dissolution medium: effect of sampling probes on dissolution rate of prednisone tablets. J. Pharm. Sci. 1982, 71 (6), 670-673.

9. Shah, V. P.; Gurbarg, M.; Noory, A.; Dighe, S.; Skelly, J. P. Influence of higher rates of agitation on release patterns of immediate-release drug products. J. Pharm. Sci. 1992, 81 (6), 500-503.

10. Lozano, R.; Joseph, J. M.; Kline, B. J. Temperature, pH and agitation rate as dissolution test discriminators of zofenopril calcium tablets. J. Pharm. Biomed. Anal. 1994, 12 (2), 173-177. 